\title{
A Man With Intractable Convulsion
}

\author{
Yun Xia, MD, *广 Anthony Cutrona, MD, *† and Timothy J. Barreiro, DO, FCCP, FACOI FACP*广t
}

\begin{abstract}
Tetanus is a rare disease in the United States. Fewer than 40 cases are reported annually because of the high incidence of vaccination. Recognition of the clinical presentations is important because laboratory recovery of pathogen is only $30 \%$, and toxin detection is rare because of consumption at motor neurons. We report a case of tetanus in an elderly man who had a reaction to tetanus vaccination as a child and was nonvaccinated through adult life.
\end{abstract}

Key Words: tetanus, convulsion, trismus, muscle spasm, muscle contraction

(Infect Dis Clin Pract 2015;23: e28-e29)

\section{CASE PRESENTATIONS}

A 67-year-old man presented to the emergency room with back and neck pain, trouble swallowing, and trismus. A welding accident 2 months previously left a healed metal burn on his left arm. One week before presentation, he had a closed fracture to his right thumb with subungual hematoma. The hematoma became infected and was treated with drainage, trimethoprimsulfamethoxazole (Bactrim DS [double strength]), and ciprofloxacin. Three days into treatment, increased right-thumb pain required repeat drainage. Symptoms progressed to back pain, neck stiffness, and dizziness and advanced to trouble swallowing and trismus, which prompted hospital admission.

A welder by trade and a current smoker, he denied alcohol or drug abuse. His medical history included hypertension, idiopathic thrombocytopenia, gastroesophageal reflux disease, lumbar spine surgery, appendectomy, and tonsillectomy. He was allergic to tetanus vaccine, penicillin, and oxymetazoline. His medications included metoprolol succinate (Toprol XL), doxycycline, prednisone, and diphenhydramine (Benadryl).

Examination revealed a normal-built man. Temperature was $98.5^{\circ} \mathrm{F}$, heart rate 98 beats $/ \mathrm{min}$, blood pressure 190/85 mm Hg, and respiratory rate 12 breaths/min. Testing revealed forceful closing of the jaw as from spasm of the masticatory muscles, nuchal rigidity, and increased muscle tone. His left thumb had a few punched holes with white matter underneath the nail (Fig. 1). Lungs were clear with no wheezing; heart and abdominal examinations were normal. Cranial nerve examination was grossly normal. Strength and light-touch sensation were normal. Reflex of the patella was hyperactive on both sides. A complete blood count revealed platelet at $84 \times 10^{9} / \mathrm{L}$. The metabolic panel and urinalysis

From the * Department of Medical Education and Research, St Elizabeth Health Center, Youngstown; †Department of Medicine, Northeast Ohio Medical University, Rootstown; and $\$$ College of Osteopathic Medicine, Ohio University Heritage, Athens, $\mathrm{OH}$

Correspondence to: Yun Xia, MD, Department of Medical Education Research, St Elizabeth Health Center, 1044 Belmont Ave, Youngstown, OH 44501. E-mail: Yun_Xia@hmis.org.

The authors have no funding or conflicts of interest to disclose.

Supplemental digital content is available for this article. Direct URL citation appears in the printed text (www.infectdis.com).

Copyright (C) 2015 Wolters Kluwer Health, Inc. All rights reserved. This is an open access article distributed under the terms of the Creative Commons Attribution-NonCommercial-NoDerivatives 3.0 License, where it is permissible to download and share the work provided it is properly cited. The work cannot be changed in any way or used commercially.

ISSN: 1056-9103 were normal. Computed tomography scans of the neck and lung both did not show abnormalities.

Over the hospital course, progressive shortness of breath, throat tightness, and change in mental status required initiation of mechanical ventilation. Lumbar puncture was normal. He developed rhythmic, tonic muscle contraction muscles and jerky movements (see Supplemental Digital Content, Video, http:// links.lww.com/IDCP/A15), but electroencephalogram showed no seizures.

With a provisional diagnosis of tetanus, infectious disease specialist was consulted. Ceftriaxone and metronidazole were started. Tetanus immunoglobulin $1000 \mathrm{U}$ was given intramuscularly, and the case was reported to the Ohio Centers for Disease Control and Prevention. Despite maximal efforts, his tonic muscle contraction was intractable, and his clinical course deteriorated. He continued to have stimulus-enhanced muscle spasms, boardlike abdomen, and autonomic instability with alternating tachycardia and bradycardia, along with rhabdomyolysis causing acute kidney injury. On day 8 , the patient died.

\section{DISCUSSION}

The term tetanos came from Greek, meaning "to stretch." Tetanus is defined as acute onset of hypertonia and painful muscle spasms without other apparent medical causes. It is caused by Clostridium tetani, an anaerobic, mobile, gram-positive rod with terminal spore. Clostridium tetani produces the hemolytic toxin tetanolysin and neurotoxin tetanospasmin, the latter referred to as tetanus toxin. Through retrograde intraneuronal transport, the toxin reaches presynaptic terminals, blocks the release of glycine and GABA ( $\gamma$-aminobutyric acid), inhibits cleavage of synaptobrevin, and thus causes tonic muscle spasms. ${ }^{1}$

Clostridium tetani is widespread in human and animal excrement. Spores of $C$. tetani are ubiquitous in soil and around the human habitation. Tetanus can be introduced into human body through deep puncture wounds where anaerobic bacterial growth is facilitated. Incubation periods range from 3 to 21 days, but averaging 8 days. In the United States, tetanus has an annual incidence 0.10 cases per million between 2001 and 2008, and the incidence of tetanus is declining. Tetanus carries a case-fatality rate of $13.2 \%$ overall and $31.3 \%$ in high-risk groups. ${ }^{2,3}$

Four basic forms of tetanus are known: neonatal, local, cephalic, and generalized, with $80 \%$ being the generalized form. In adult patients, typical presentation includes nuchal rigidity, apnea, dysphagia, and generalized spasms; with the most characteristic sign of generalized tetanus being lockjaw or trismus. The diagnosis of tetanus is mostly based on clinical manifestations, rather than bacteriologic findings. ${ }^{4}$ The organisms can be recovered in only $30 \%$ of cases, and positive culture can occur in immunized, healthy people. ${ }^{5}$ Electromyographic test can show continuous discharge of motor units and shortening or absence of silent interval after action potential. Creatine kinase usually is elevated.

The 3 objectives of management of tetanus are (1) to neutralize unbound tetanospasmin, (2) to halt the production of toxin, (3) and to provide supportive care. Patient should receive active immunization with tetanus vaccine and passive immunization with tetanus immune globulin (TIG). Passive immunization with TIG does not affect the neuron-bound toxin, but it can neutralize 


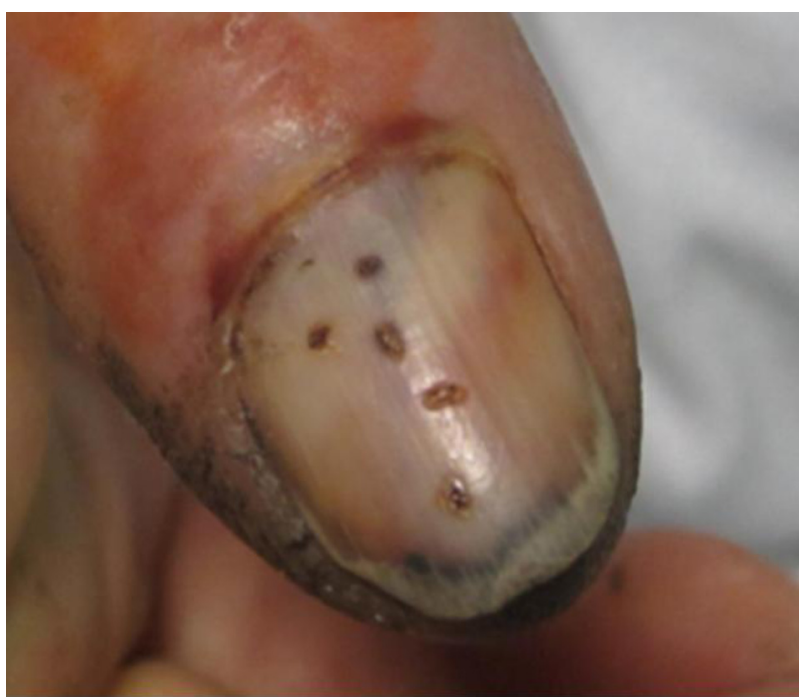

FIGURE 1. Photograph of the left thumb shows a few punched holes with white matter underneath the nail.

free tetanospasmin to prevent its binding to the neuronal synapse. In suspected tetanus, tetanus-prone wounds, and injuries associated with incomplete vaccination or unknown vaccination status, the use of TIG is recommended. ${ }^{4}$ The dose is $500 \mathrm{U}$ of TIG from human origin or 1500 to $5000 \mathrm{U}$ from TIG from animal origin. ${ }^{6}$

Antibiotics and elimination of the anaerobic environment with aeration and wound debridement can reduce tetanus toxin. Clostridium tetani is sensitive to $\beta$-lactams, macrolides, tetracycline, and metronidazole. Metronidazole is the preferred antimicrobial because of its better penetration in anaerobic tissues; it can be given at doses of $500 \mathrm{mg}$ every 6 hours intravenously or orally for 10 to 14 days. $^{7}$

Supportive care, including control of muscle spasms and autonomic stability, may be achieved with various ways. It is paramount to protect airway and have the patient rest in a dark, quiet room to minimize sensory stimulations. Benzodiazepines are often preferred drugs because they not only control the rigidity and spasms but also sedate the patients. The neuromuscularblocking agents, such as vecuronium and pancuronium, are used when benzodiazepines are insufficient. Baclofen, a GABA agonist, can also be used intrathecally to control spasms and reduce the need for tracheal intubation. Other agents such as dantrolene, barbiturates, propofol, and chlorpromazine can also be used.

Complications of tetanus, as the case with our patient, include autonomic instability and rhabdomyolysis with renal failure. ${ }^{8,9}$ Excessive catecholamine release can cause hypertension and tachycardia alternating with bradycardia and hypotension. Medications that lead to adrenergic blockade and inhibition of autonomic reactivity are the proper management to achieve control of autonomic function. Magnesium sulfate, which blocks catecholamine release and desensitizes catecholamine receptors, can be used alone or with benzodiazepine. It can be started with an intravenous loading dose $5 \mathrm{~g}$ (or $75 \mathrm{mg} / \mathrm{kg}$ of body weight), followed by 2 to $3 \mathrm{~g} / \mathrm{h} .{ }^{10}$ Other alternatives include labetalol, esmolol, and clonidine. Prolonged contractures and convulsions may result in bone fractures and rhabdomyolysis with renal failure. Adequate fluid resuscitation and early nutritional support are imperative because of high metabolism state from constant muscle contracture.

\section{SUMMARY}

The diagnosis of tetanus poses a challenge because it is rarely seen now. The diagnosis is made mainly on clinical manifestations. Practitioners should carefully evaluate patients with muscle spasm and include tetanus in the differential diagnoses. Treatment includes active and passive immunizations, antibiotics, wound debridement, antispastic agents, and supportive care. It is paramount to follow the current immunization recommendations to prevent this highly lethal disease.

\section{REFERENCES}

1. Bleck TP. Tetanus. In: Scheld WM, Whitley RJ, Durack DT, eds. Infections of the Central Nervous System. New York: Raven Press; 1991:603-624.

2. Mariely Otero-Maldonado M, Bosques-Rosado M, Soto-Malavé R, et al. Tetanus is still present in the 21st century: case report and review of literature. Asoc Med Puerto Rico. 2011;103(2):41-57.

3. Centers for Disease Control. Tetanus surveillance. Morb Mortal Wkly Rep. 2011;60:365-369.

4. Peter A, David M, Shagufa A. Tetanus: a review. South Med J. 2011;104: 613-617.

5. Tetanus. In: Epidemiology and Prevention of Vaccine. Preventable Diseases. The Pink Book. 11th ed. Atlanta, GA: National Center for Immunization and Respiratory Diseases, Centers for Disease Control and Prevention; 2009:273-282.

6. Afshar M, Raju M, Ansell D, et al. Narrative review: tetanus - a health threat after natural disasters in developing countries. Ann Intern Med. 2011;154:329-335.

7. Joshi S, Agarwal B, Malla G, et al. Complete elimination of tetanus is still elusive in developing countries: a review of adult tetanus cases from referral hospital in eastern Nepal. Kathmandu Univ Med J. 2007;5: 378-381.

8. Gibson K, Bonaventure Uwineza J, Kiviri W, et al. Tetanus in developing countries: a case series and review. Can J Anaesth. 2009;56:307-315.

9. Martinelli R, Matos CM, Rocha H. Tetanus as a cause of acute renal failure: possible role of rhabdomyolysis. Rev Soc Bras Med Trop. 1993;26:1-4.

10. Attygalle D, Rodrigo N. Magnesium as first line therapy in the management of tetanus: a prospective study of 40 patients. Anaesthesia. 2002;57:811-817 